\title{
Demonstration of a novel III-V-on-Si distributed feedback laser
}

\author{
S. Keyvaninia ${ }^{1}$, G. Roelkens ${ }^{1}$, R. Baets ${ }^{1}$, F. Lelarge ${ }^{2}$, G-.H. Duan ${ }^{2}$, S. Messaoudene ${ }^{3}$, J.-M. Fedeli ${ }^{3}$, \\ D. Van Thourhout ${ }^{1}$ \\ ${ }^{1}$ Photonics Research Group, INTEC, Ghent University-IMEC, B-9000 Gent, Belgium \\ ${ }^{2}$ III-V Lab, a joint lab of 'Alcatel-Lucent Bell Labs France', 'Thales Research and Technology' and 'CEA Leti', \\ Campus Polytechnique, 1, Avenue A. Fresnel, 91767 Palaiseau cedex, France \\ ${ }^{3}$ CEA-LETI, Minatec Campus, 17 Rue des Martyrs, 38054 Grenoble, France
}

\begin{abstract}
We present the first experimental demonstration of a novel III-V-on-silicon distributed feedback laser type. All structures defining the laser cavity are defined in the silicon. Alasing threshold of 50mA and a side mode suppression ratio higher than $40 \mathrm{~dB}$ is achieved.
\end{abstract}

OCIS codes: (250.0250) Optoelectronics; (250.5300) Photonic integrated circuits; (250.5960) Semiconductor lasers.

\section{Introduction}

Silicon-On-Insulator (SOI) is gaining interest as a novel platform for integrated optical circuits. Its large refractive index contrast allows for ultra-compact devices but the interest in this technology stems mostly from the expectation that the maturity and low cost of CMOS-technology can be applied for advanced photonics products. However, the integration of a laser on this platform is hampered by silicon's indirect bandgap. Building light sources, and in particular laser sources, on integrated silicon circuits is a big challenge. In that sense the heterogeneous integration of III-V/silicon lasers combines the best of two worlds. In spite of the rapid progress in this technology since about 2006, the design and fabrication of hybrid silicon lasers present specific challenges and trade-offs. There are many choices to be made, both in terms of the cavity structure, the optical coupling between the silicon waveguide and the III-V waveguide and the technological approach. A relatively large variety of device approaches have been reported in the past years [1]. Two main technologies are used to heterogeneously integrate III-V epitaxial layer stacks on a silicon waveguide circuit: molecular bonding and adhesive bonding. We focus on an adhesive die-to-wafer and wafer-to-wafer bonding process, based on DVS-BCB as a bonding agent, given more relaxed requirements on the III-V wafer surface quality (contamination, particles, roughness). This principle has proven to work in several different configurations [1][2]. A promising method to achieve a high performance laser cavity is to use an intracavity double taper structure, using taper-based mode transformers in both the III-V and silicon waveguides [2]. However, the fabrication of taper-based mode converters is not straightforward because of the narrow tips required and the strong dependence of the performance on alignment accuracy. In this paper we propose a new approach to obtain a compact, efficient and single mode hybrid silicon laser, based on an active DBR grating which requires no complex coupling structures such as narrow-tip tapers in the III-V process.

(a)

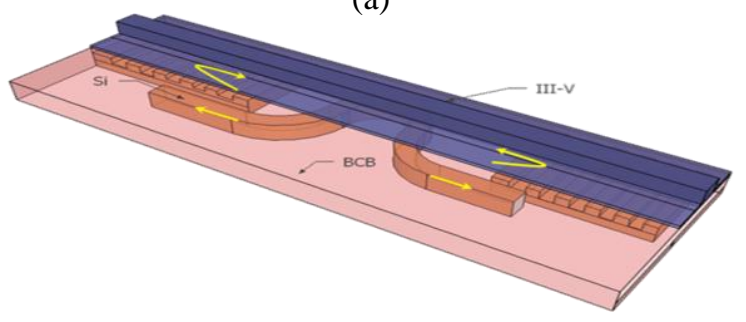

(b)

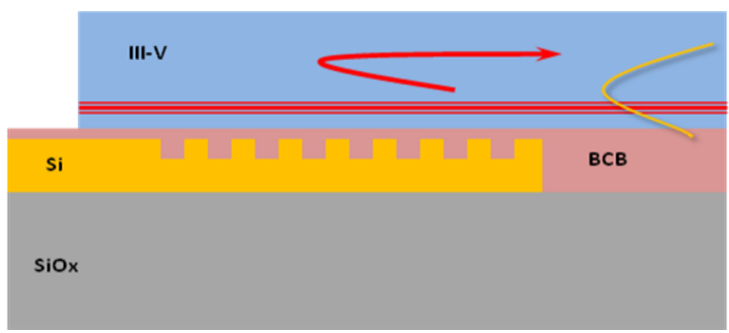

ig. 1. (a) Schematic of the active DBR with the evanescent coupling waveguide in the center of the laser cavity (b) schematic view of a hybrid DBR reflector in the hybrid silicon laser.

\section{Laser cavity design}

The hybrid platform consists of an SOI substrate with a slab of III-V material bonded on top and a thin bonding layer (DVS-BCB) in between. An optimal laser cavity design should consist of a section where the optical mode is 
completely confined to the III-V waveguide layer, while the wavelength selective feedback is provided by structures defined in the silicon, thereby taking advantage of the resolution and accuracy of lithography tools in CMOS fabs. Of course, the laser emission should also be coupled efficiently into the silicon waveguide layer. It would thus be beneficial to think of cavities that do not use an adiabatic taper to couple light between the III-V layer and the silicon waveguides. Besides using resonant cavities in the silicon [3], this can be realized in a DBR-based cavity by using two active DBR gratings with nearly $100 \%$ reflection to define the laser cavity and where an evanescent coupling interface is positioned within the laser cavity as the laser output as shown in figure 1 . This hybrid laser design offers much simpler device fabrication and helps to reduce the device footprint.

\section{Simulation Results}

We have simulated the use of DBR reflectors extensively in the context of these hybrid silicon lasers. In a crosssectional view of the structure, two waveguides can be identified: one in the silicon DBR grating and one in the active layer. In between them there is the bonding layer (DVS-BCB), as shown in Fig 1(b). The III-V region used in the simulation has a multiple quantum well $(\mathrm{QW})$ double heterostructure, which consists of a $\mathrm{p}$-InGaAs contact layer, a p-InP cladding, a quantum well region, and an n-InP bottom cladding layer. The QW region consists of six $8 \mathrm{~nm}$ thick InGaAsP (1.55um bandgap wavelength) well layers separated by $10 \mathrm{~nm}$ thick $\mathrm{InGaAsP}$ (Eg=1.17um bandgap wavelength) barrier layers. The thickness of both SCH layers is $100 \mathrm{~nm}$ and the thickness of the n-InP and p-InP layers are $200 \mathrm{~nm}$ and $1500 \mathrm{~nm}$ respectively. The silicon rib grating has a height of $400 \mathrm{~nm}$, an etch depth of $180 \mathrm{~nm}$ and a width of $3 \mu \mathrm{m}$ for the DBR parts. Simulations were performed for the cavity reflectors and the coupling section. A 2D simulation based on the open-source eigenmode solver CAMFR [4] to calculate the first-order to firstorder mode reflection of the active waveguide is shown in figure 2(a), showing the power reflection as a function of the number of periods and the DVS-BCB thickness, for a grating period of $240 \mathrm{~nm}$ (for Bragg reflection at $1.55 \mu \mathrm{m}$ ), $50 \%$ duty cycle and an etch depth of $180 \mathrm{~nm}$. The coupling constant $\kappa$ for two different silicon waveguide heights and two different grating etch depths versus DVS-BCB thickness is plotted in figure 2 (b).
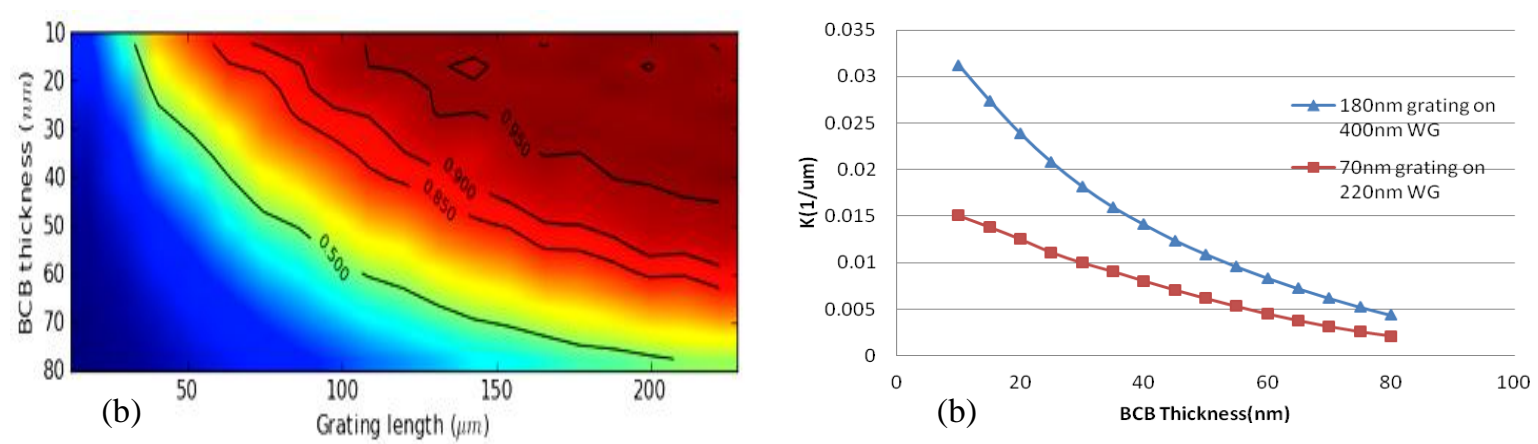

Fig. 2. (a) Grating reflection as a function of the number of grating periods and DVS-BCB thickness (b) Coupling constant $\kappa$ for a hybrid grating simulated for different grating cross-sections versus DVS-BCB thickness.

\section{Fabrication and measurement results}

The SOI substrate (200 mm wafer manufactured by SOITEC) is composed of a $400 \mathrm{~nm}$ mono-crystalline silicon layer on top of a $2 \mu \mathrm{m}$ thick buried oxide layer on a silicon substrate. The fabrication process begins with the processing of the SOI wafer incorporating a $400 \mathrm{~nm}$ thick silicon waveguide layer in a CMOS pilot line. The silicon rib waveguides have a height of $400 \mathrm{~nm}$ and an etch depth of $180 \mathrm{~nm}$. Also the DBR-gratings defining the laser cavity were etched $180 \mathrm{~nm}$ deep in the $400 \mathrm{~nm}$ thick silicon layer and had a period of $240 \mathrm{~nm}$. Afterwards, a $\mathrm{SiO}_{2}$ cladding layer was deposited and the wafer was planarized using chemical mechanical polishing (CMP) and an extra wet etching step to reach the silicon waveguide level before bonding. The III-V membrane layer is bonded onto the silicon waveguide circuit using an adhesive BCB die-to-wafer bonding approach [2]. The III-V region consists of the layers as described in the simulation section. After bonding and InP substrate removal, a SiNx hard mask was defined using $320 \mathrm{~nm}$ UV contact lithography. ICP etching was used to etch through the InGaAs layer, the InP pdoped layer and the MQW layer using $\mathrm{CH} 4: \mathrm{H} 2$. Figure 3 shows a microscope image of the fabricated device and a scanning electron microscope (SEM) picture of the coupling section in the silicon layer. The active waveguide is encapsulated with DVS-BCB. GeAu/Ni and Ti/Pt/Au contacts were used for the $\mathrm{n}$ and $\mathrm{p}$-sides respectively. In this first electrically pumped prototype, the silicon DBR-mirrors were $2 \mu \mathrm{m}$ wide, had a period of $240 \mathrm{~nm}$ and were etched $180 \mathrm{~nm}$ deep in a $400 \mathrm{~nm}$ high rib waveguide. Each mirror was $350 \mu \mathrm{m}$ long and both mirrors were separated from each other by $120 \mu \mathrm{m}$. In the middle between both mirrors we inserted a compact wedge type outcoupling 
section in the silicon layer. The light from the laser cavity is coupled out through evanescent coupling and by choosing the coupling length the output power can be engineered. The III-V waveguide above is $2.5 \mu \mathrm{m}$ wide and depending on the bonding thickness and design can be from $400 \mu \mathrm{m}$ to $800 \mu \mathrm{m}$ long. This gives enough design flexibility for optimizing the laser for the best power efficiency.

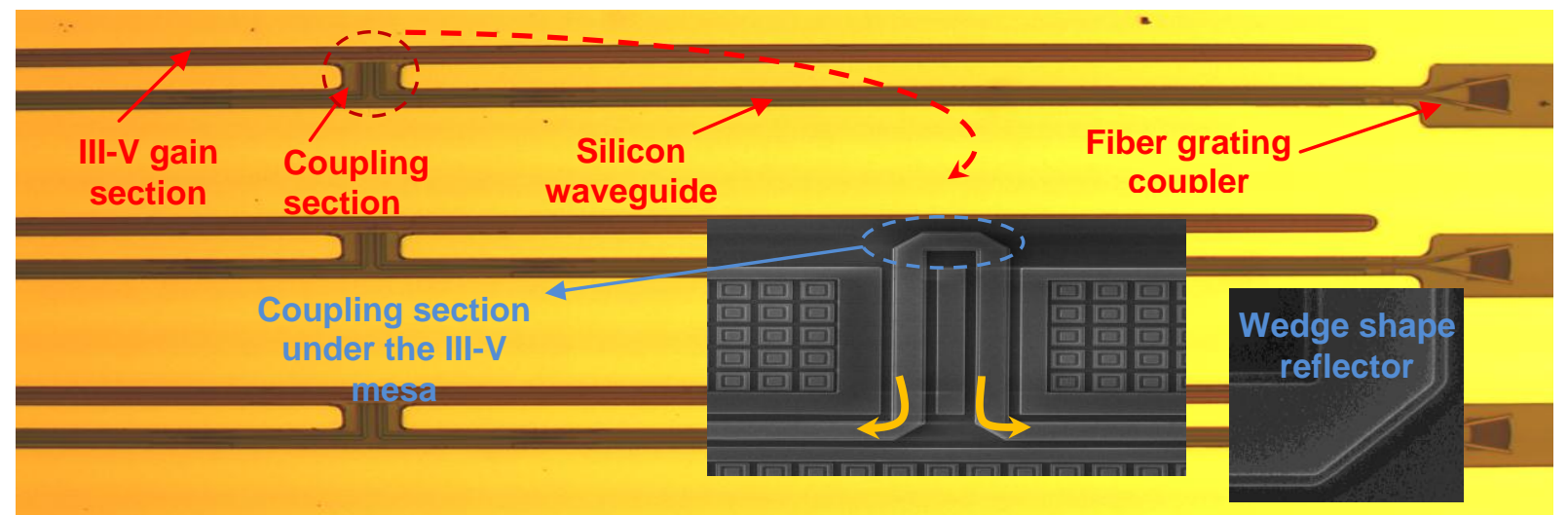

Fig. 3. Microscope picture of the fabricated lasers, showing the coupling section, the III-V integration on silicon (after n-type metallization) and the passive silicon output grating couplers.

In this paper we are presenting the results for a III-V laser of $700 \mu \mathrm{m}$ length. Single wavelength operation is observed with side mode suppression ratio consistently around $40 \mathrm{~dB}$. The device has a lasing threshold of 50mA and a maximum fiber coupled power of $-25 \mathrm{dBm}$ at both sides in continuous wave (figure 4). Huge losses due to an error in the fabrication of the wedge corner (almost $6 \mathrm{~dB}$ loss per 45 degree facet) and $10 \mathrm{~dB}$ loss due to the chip to fiber grating coupler and a larger than expected bonding layer thickness (in this demonstration 120nm) reduce the coupling efficiency substantially. Outcoupling will be significantly improved by reducing the bonding layer and replace the wedge corner by a bend coupling section, which will result in mW-level output power [6].
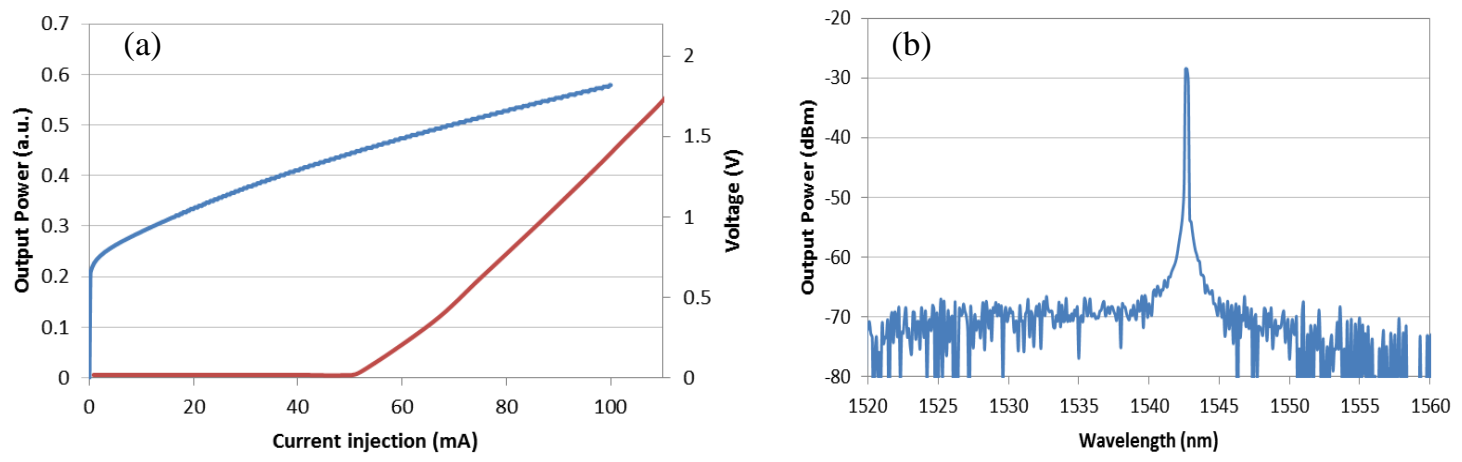

Fig. 4. (a) The L-I-V curve for a single mode laser ;(b) Lasing spectrum of the hybrid single mode laser for $80 \mathrm{~mA}$ current injection at $20^{\circ} \mathrm{C}$

\section{Conclusion}

We present the first experimental demonstration of a new concept for the realization of III-V-on-silicon hybrid lasers, providing high optical confinement in the III-V waveguide layer, while the wavelength selective feedback elements are implemented in the silicon. This opens up a route towards the large volume realization of single mode lasers with precisely defined emission wavelength on a silicon waveguide platform.

\section{References}

[1] G. Roelkens, L. Liu, et al., Laser Photonics Rev. P.DOI: 10.1002/lpor.200900033 (2010).

[2] M. Lamponi, S. Keyvaninia, et al., "Low-Threshold Heterogeneously Integrated InP/SOI Lasers With a Double Adiabatic Taper Coupler," IEEE Photon. Technol. Lett. 24, 76-78 (2012).

[3] Y. De Koninck, G. Roelkens and R. Baets "Demonstration of a novel single-mode hybrid silicon microlaser" proceedings of the annual meeting of the IEEE Photonics society 2012

[4] CAMFR ( http://camfr.sourceforge.net)

[5] S. Keyvaninia, M. Muneeb, et al., "Multiple die-to-wafer adhesive bonding for heterogeneous integration ," presented at the 16th European Conference on Integrated Optics, Barcelona, Spain, April 2012

[6] S. Keyvaninia, Y. De Koninck, et al., "A novel III-V-on-Si distributed feedback laser design," presented at the 16th European Conference on Integrated Optics, Barcelona, Spain, April 2012 